\title{
Novel Core-shell Nanoscale Precipitates in High Performance PbSe-CdSe Thermoelectric Materials
}

\author{
Songting Cai, Xiaobing Hu, Mercouri Kanatzidis and Vinayak Dravid \\ Northwestern University, Evanston, Illinois, United States
}

Thermoelectric (TE) devices can convert between thermal and electrical energy directly and reversibly, which are considered as a promising energy-saving technology [1]. The conversion efficiency of TE devices requires low thermal conductivity over the entire working temperature range. Construction of embedded nanoscale second phase in the bulk matrix is an efficient strategy to reduce lattice thermal conductivity, since the induced lattice strain can effectively scatter more heat-carrying phonons. With judiciously chosen phases and appropriate heat treatment, one can optimize the strain level at the interface by control of the second phase's shape, size, distribution and their coherency with the matrix. Therefore, it is of high importance to reveal the interfacial details in nanostructured thermoelectric materials to better understand the underlying mechanisms [2]-[3]. Here, we have characterized endotaxial CdSe nanoscale precipitates with core-shell architecture in $\mathrm{PbSe}$, which are responsible for significant reduction in lattice thermal transport [4].

Figure 1(a) is a typical high-angle annular dark field STEM (HAADF-STEM) image of the PbSe$10 \%$ CdSe sample. A large amount of faceted precipitates $\sim 50-200 \mathrm{~nm}$ can be observed within the PbSe matrix. The contrast variation verifies that the precipitates have a tetrahedral shape. All the edges of the precipitates in one grain are parallel to each other, indicating a preferred growth plane as well as coherency between the two phases. Energy dispersive spectroscopy (EDS) mappings further confirms that the second phases are rich in $\mathrm{Cd}$ and severely deficient in $\mathrm{Pb}$, Figure 1 (b).

From the bright field TEM image shown in Figure 2 (a), two $20 \mathrm{~nm}$ thick bands are observed to sandwich between the interfaces of matrix and main body of the precipitate, constructing a core-shell architecture. The selected area diffraction pattern (SAED) in Figure 2 (b) confirms that the main brightest spots belong to rock-salt $\mathrm{PbSe}$ and zincblende CdSe phases along the [110] zone axis. On the contrary, the more complicated streaking and the two sets of extra spots observed along $\{111\}$ planes of the matrix belong to the hexagonal wurtzite CdSe phase along the [100] zone axis.

For further atomic-scale analysis, we employed aberration corrected (probe) high-resolution STEM imaging. Figure 2(c) is a typical HAADF image, revealing an endotaxial precipitation nature. According to the zoom-in versions shown in Figure 2(d)-(g), the atom stacking changes from ABC/ABC at the solid solution and precipitate core to $\mathrm{AB} / \mathrm{AB}$ in the hexagonal wurtzite phase at the shell. In addition, several stacking faults can be observed within the shell.

Consequently, the novel core-shell nanostructure leads to both significant reduction in lattice thermal conductivity and a record $Z T_{\text {ave }}$ of $\sim 0.83(400-923 \mathrm{~K})$, the highest report value in p-type, Te-free PbSe systems.

The presentation will cover how advanced electron microscopy provides effective toolset for qualitative and quantitative interface/defects analyses in energy materials broadly. Specifically, the ongoing research sheds light on optimization of heat treatment design and kinetics in thermoelectric materials based on the nanoscale understanding of the microstructure [5]. 


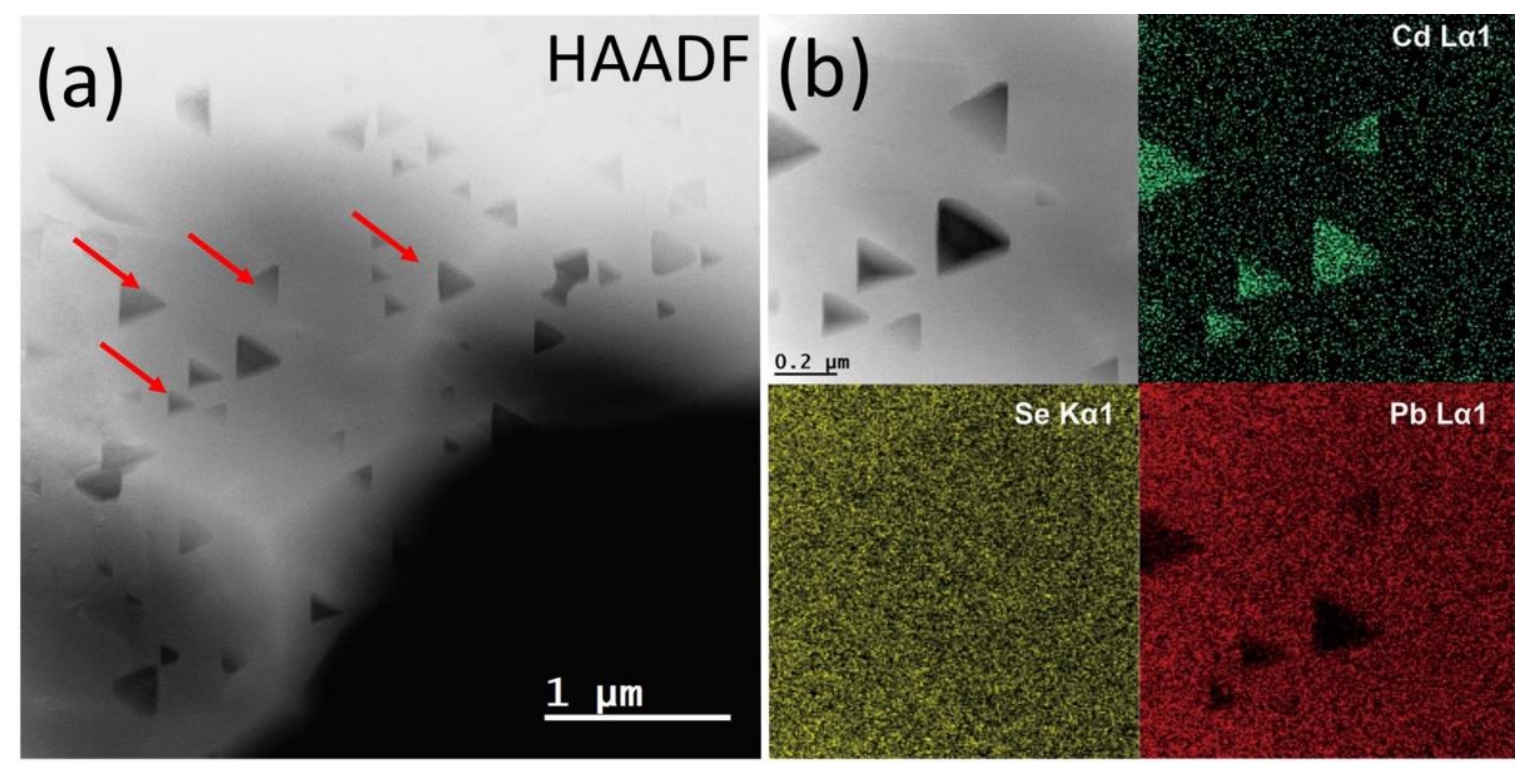

Figure 1. Figure 1. Scanning/transmission electron microscopy (S/TEM) analyses of PbSe-10\%CdSe. (a) High-angle annular dark field image. A large amount of triangular nano-precipitates is embedded within the grains of the matrix. (b) EDS mappings of selected area in (a) [4].

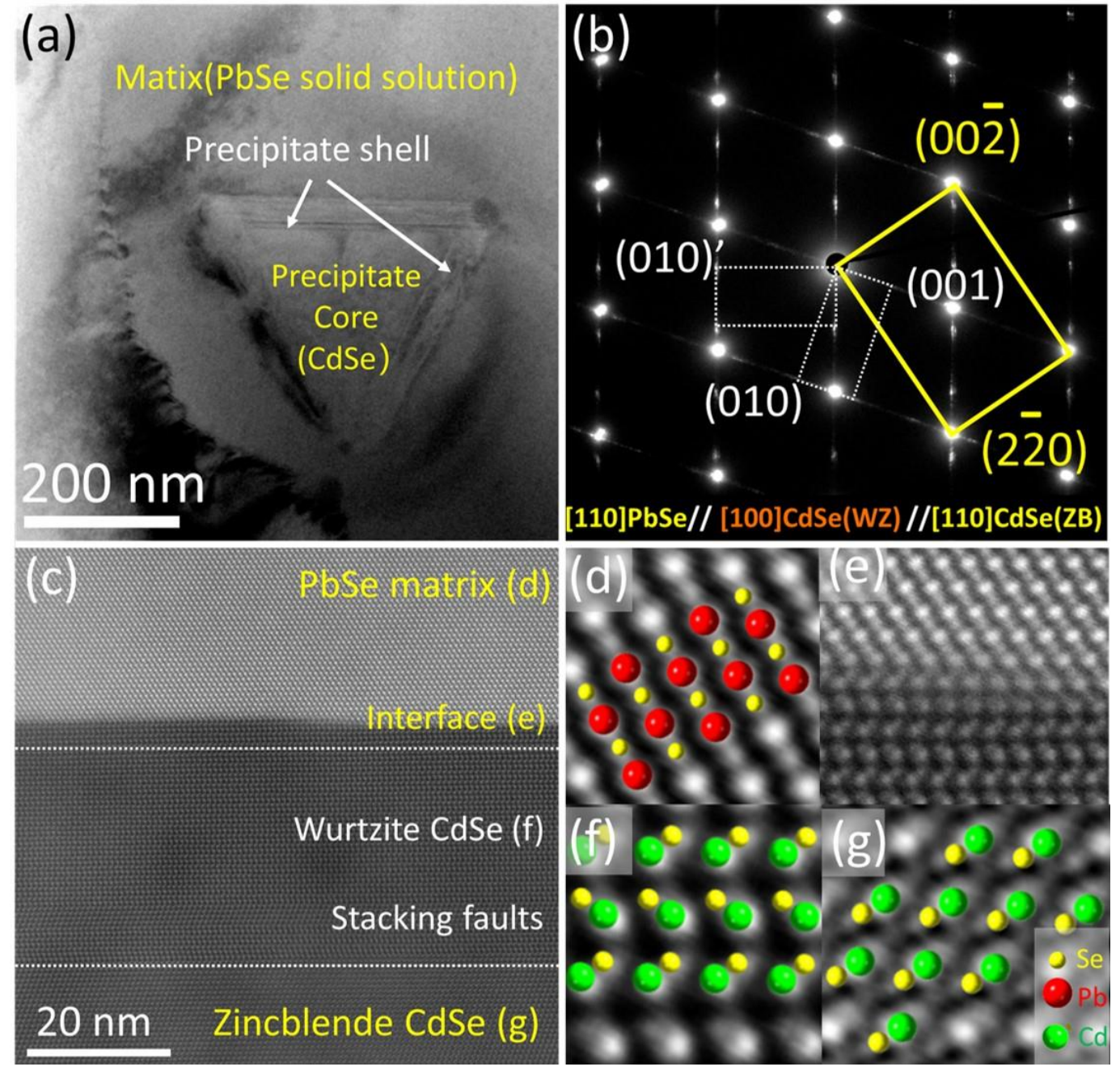


Figure 2. Figure 2. (a) Typical TEM image of a precipitate sitting in the matrix. Diffraction contrast highlights three different regions: the matrix, the main body of the precipitate and $\sim 20 \mathrm{~nm}$ thick layers as shells between the interface. (b) Selected area diffraction pattern (SAED) taken along [110] zone axis of the matrix, revealing that the interface is wurtzite CdSe phase. (c) High-resolution STEM image (HRSTEM) with HAADF mode image of one edge of the precipitate and the matrix. The atomic stacking of the matrix, matrix-shell interface, the shell, and the core of the precipitate are highlighted in $(\mathrm{d})-(\mathrm{g})$, respectively [4].

\section{References}

[1] Zeier, W. G. et al. Thinking Like a Chemist: Intuition in Thermoelectric Materials. Angewandte Chemie International Edition 55, 6826-6841 (2016).

[2] He, J., Kanatzidis, M. G. \& Dravid, V. P. High performance bulk thermoelectrics via a panoscopic approach. Materials Today 16, 166-176 (2013).

[3] Tan, G., Zhao, L.-D. \& Kanatzidis, M. G. Rationally Designing High-Performance Bulk Thermoelectric Materials. Chemical Reviews 116, 12123-12149 (2016).

[4] Cai, S. et al. Discordant nature of Cd in PbSe: off-centering and core-shell nanoscale CdSe precipitates lead to high thermoelectric performance. Energy \& Environmental Science 13, 200-211 (2020).

[5] This work was supported by the Department of Energy, Office of Science Basic Energy Sciences under grant DE-SC0014520, DOE Office of Science. This work made use of the EPIC facility of Northwestern University's NUANCE Center, which has received support from the Soft and Hybrid Nanotechnology Experimental (SHyNE) Resource (NSF ECCS-1542205); the MRSEC program (NSF DMR-1720139) at the Materials Research Center; the International Institute for Nanotechnology (IIN); the Keck Foundation; and the State of Illinois, through the IIN. User Facilities are supported by the Office of Science of the U.S. Department of Energy under Contract No. DE-AC02-06CH11357 and DE-AC02-05CH11231. 